\title{
Humic Substance Fractions and Attributes of Histosols and Related High-Organic-Matter Soils from Brazil
}

\author{
Gustavo Souza Valladares \\ Embrapa Satellite Monitoring, Campinas, Brazil \\ Marcos Gervasio Pereira and Lúcia Helena Cunha dos Anjos \\ Soils Department, Federal Rural University of Rio de Janeiro, \\ Seropédica, Brazil \\ Vinícius de Melo Benites \\ Embrapa Soils, Rio de Janeiro, Brazil \\ Adierson Gilvani Ebeling \\ Soils Department, Federal Rural University of Rio de Janeiro, \\ Seropédica, Brazil \\ Rondinele de Oliveira Mouta \\ PUC Chemistry, Rio de Janeiro, Brazil
}

\begin{abstract}
Knowledge of the distribution of soil organic matter (SOM) fractions is important in managing soils toward a sustainable agricultural system in a tropical environment. However, data on Histosols is limited. This study developed 19 profiles of Histosols and soils with high organic-matter content from different regions of Brazil. Soil organic matter was fractionated into fulvic acids (FAF), humic acids (HAF), and humin (HUM). The ratios HAF/FAF and AE (alkaline extract)/HUM were calculated. The objectives were to evaluate the method for SOM fractionating in Histosols and related soils and to correlate the distribution of organic fractions with other soil attributes. The humic fractions presented significant
\end{abstract}

Received 18 January 2005, Accepted 22 September 2006

Address correspondence to Marcos Gervasio Pereira, Soils Department, UFRRJ, Federal Rural University of Rio de Janeiro, BR 465, Km 7, 23890-000, Seropédica (RJ), Brazil. E-mail: gervasio@ufrrj.br 
each fraction plays a role in the mechanisms (Ferreira et al. 2002; Jones and Huang 2003; Lal 2004)

Because of the different functions performed by the humic fractions and the differentiated characteristics of Histosols and related soils with high organic-matter content, these attributes should be better studied in these soils. Knowledge about soil organic-matter fractions is important to manage the chemical and physical properties toward a sustainable agricultural system, especially in a tropical environment.

The objectives of this study were to evaluate organic-matter fractionation method in humic substances applied to Histosols and related soils with high organic-matter content. and to correlate the humic fraction distribution and 
correlations with other soil attributes, the best being the correlation between FAF and nutrient level. The HAF and HUM presented high correlation with cationic exchange capacity, active acidity $\left(\mathrm{H}^{+}\right)$and $\mathrm{pH}$. Humin and the alkaline extract absorbance measured at $380 \mathrm{~nm}$ and $465 \mathrm{~nm}$ and presented good correlation with total organic carbon.

Keywords: Organic matter, peat soils, soil chemical properties, tropical soils

\section{INTRODUCTION}

The central concept of Histosols is that of soils forming from organic soil materials. Thus, the development of methods for organic matter characterization is more important than for mineral soils. The different humic fractions, which are higher in the Histosols soil order because of its predominantly organic nature, should be characterized to estimate the soil inherent tendency to mineralization and susceptibility to subsidence and loss of soluble organic matter. In tropical climates, the approach of fractionating humic substances in Histosols and relating them to soil attributes is even more relevant to sustainable agriculture.

The definition of humic substances (HS) is not simple and reflects the organic matter complexity. Humic substances may be defined as a series of yellow-brown and black polymers of relatively high molecular weight and formed by secondary, biotic, and abiotic synthesis reactions (Stevenson 1994). However, as explained by MacCarthy (2001), because of the uncertain aspect of this and other HS definitions, it is also usual to define these materials operationally in terms of laboratory procedures used to extract them from soils, sediments, and waters. The classic procedure to extract humic substances from soils results in three main fractions: humic acids (HAF), fulvic acids (FAF), and humin compounds (HUM). These fractions are defined in terms of their solubility in aqueous medium depending on the $\mathrm{pH}$ of the extracting solution (Schnitzer and Khan 1978; Tombácz and Meleg 1990). The alkaline solutions, most often $0.1 \mathrm{~mol} \mathrm{~L}^{-1}$ $\mathrm{NaOH}$, extract HAF and FAF from soil, leaving the HUM associated to the mineral phase. The acidification of the black-colored alkaline extract results in the HAF coagulation (black or brownish precipitate), whereas the FAF remains soluble (yellow-brownish solution). Although this separation procedure seems artificial, some segregation degree of polymeric materials with different chemical properties is achieved (McBride 1994).

None of the soil organic matter fractions isolated represent separation of individual compounds but rather a mixture of heterogeneous compounds with similar chemical behavior. The humic substances play an important role in the flow of nutrients in ecological systems, in carbon $(\mathbf{C})$ emission into the atmosphere, and in interactions with heavy metals and pesticides, where 
each fraction plays a role in the mechanisms (Ferreira et al. 2002; Jones and Huang 2003; Lal 2004)

Because of the different functions performed by the humic fractions and the differentiated characteristics of Histosols and related soils with high organic-matter content, these attributes should be better studied in these soils. Knowledge about soil organic-matter fractions is important to manage the chemical and physical properties toward a sustainable agricultural system, especially in a tropical environment.

The objectives of this study were to evaluate organic-matter fractionation method in humic substances applied to Histosols and related soils with high organic-matter content, and to correlate the humic fraction distribution and ratios with some attributes of these soils.

\section{MATERIAL AND METHODS}

Nineteen soil profiles from different Brazilian edafoclimatic regions (Table 1) were collected, being 17 profiles of Histosols, one Inceptisol, and one Entisol profile, the last two having high-organic-matter content in the surface horizons. The soils were collected, described, and characterized according to procedures in the Manual for Soil Description and Collecting (Lemos and Santos 1996) and the Soil Survey Manual (Soil Survey Division Staff 1993). The profiles were classified according to the Brazilian soil classification system, SiBCS (Embrapa 1999) and the soil taxonomy system (Soil Survey Staff 1999) (Table 1).

The soil chemical and physical properties were analyzed according to Embrapa (1997, 1999). The humic substances fractionating was performed in triplicates in 53 horizon samples from 19 soil profiles (Table 2), according to differential solubility techniques, using the humic fractions concept established by the Humic Substances International Society, as adapted by Benites, Madari and Machado (2003). Soil samples containing about $40 \mathrm{mg}$ of $\mathrm{C}$ (in the Histosols, the values ranged from 0.1 to $0.5 \mathrm{~g}$ of soil sample) and $25 \mathrm{~mL}$ of $0.1 \mathrm{~mol} \mathrm{~L}^{-1} \mathrm{NaOH}$ were used in the extraction of HAF and FAF, with a contact time of $24 \mathrm{~h}$. The separation between the alkaline extract and the residue was performed by centrifugation at $10,000 \mathrm{~g}$ (gravity) for $20 \mathrm{~min}$. Two residue washings were carried out with $19 \mathrm{~mL}$ of the same solution by adding the extracts previously reserved that resulted in a final volume of approximately $45 \mathrm{~mL}$. The residue was collected and reserved for the determination of carbon as humin compounds (HUM). The alkaline extract $(\mathrm{AE})$ had the $\mathrm{pH}$ lowered to $2.0 \pm 0.1$ with $20 \%$ sulfuric acid $\left(\mathrm{H}_{2} \mathrm{SO}_{4}\right)$ and decanted for $18 \mathrm{~h}$. The $\mathrm{H}_{2} \mathrm{SO}_{4}$ solution was used instead of hydrochloric acid $(\mathrm{HCl})$ to avoid interference from the chloride anion in the $\mathrm{C}$-determination procedure by the titulometric method with dichromate. The precipitate, HAF, was separated from the soluble fraction by means of centrifugation at $3,000 \mathrm{~g}$ (gravity) for $5 \mathrm{~min}$, rediluted in $0.1 \mathrm{~mol} \mathrm{~L}^{-1}$ 
sodium hydroxide $(\mathrm{NaOH})$ solution, and the volume was taken to $50 \mathrm{~mL}$. The soluble portion in the acidified extract, fulvic acid fraction (FAF), had the volume also adjusted to $50 \mathrm{~mL}$ using distilled water.

The $\mathrm{C}$ determination in the HAF and FAF extract was performed according to the method of Yeomans and Bremner (1998) using 1 to $5 \mathrm{~mL}$ of extract, $1 \mathrm{~mL}$ of $0.2 \mathrm{~mol}_{\mathrm{c}} \mathrm{L}^{-1}$ potassium dichromate $\left(\mathrm{K}_{2} \mathrm{Cr}_{2} \mathrm{O}_{7}\right)$, and $5 \mathrm{~mL}$ of concentrated $\mathrm{H}_{2} \mathrm{SO}_{4}$. The $\mathrm{K}_{2} \mathrm{Cr}_{2} \mathrm{O}_{7}$ concentrations were adjusted so that 10 to $75 \%$ of the oxidizer was consumed in the reaction, thus maintaining the titration within the correlation linear range with C content (Nelson and Sommers 1982). Carbon content was computed by the following equation:

$$
\mathrm{g} \mathrm{C} \mathrm{kg}^{-1} \text { soil }=\frac{\left(\mathrm{mmol}_{\mathrm{c}} \mathrm{L}^{-1} \mathrm{Cr}_{2} \mathrm{O}_{7}^{2-}-\mathrm{mmol}_{\mathrm{c}} \mathrm{Fe}^{2+}\right) 0.03 .100}{\text { mass of sample }(\mathrm{g})}
$$

The HAF/FAF ratio as the soluble fractions in the alkaline extract $(\mathrm{AE}=$ fulvic acid fraction + humic acid) and the humin compounds (AE/ HUM) were then calculated. The total $\mathrm{C}\left(\mathrm{C} \_\mathrm{CHN}\right)$ and total $\mathrm{N}$ were determined by dry combustion of soil samples with $5.0 \mathrm{mg}( \pm 0.1 \mathrm{mg})$. A Perkin Elmer $2400 \mathrm{C}-\mathrm{H}-\mathrm{N}-\mathrm{S}$ elemental analyzer was used as the reference method.

To obtain optical absorbance values of the alkaline extracts, soil samples containing $100 \mathrm{mg}$ of organic $\mathrm{C}$ were weighed and set in contact with $100 \mathrm{~mL}$ of $0.1 \mathrm{~mol} \mathrm{~L}^{-1} \mathrm{NaOH}$ solution for $24 \mathrm{~h}$. After that, the soil suspension was filtered and the extract diluted in the proportion of 1:5 with distilled water. The color of the solution was read in a colorimeter at wavelengths of 380 , 465 , and $665 \mathrm{~nm}$.

The bulk density was calculated from the weight of the oven-dry mass and volume of the soil core. For chemical analyses, samples of the fine earth (soil $<2 \mathrm{~mm}$ ) were extracted with $1 \mathrm{~mol} \mathrm{~L}^{-1}$ potassium chloride $(\mathrm{KCl})$ for calcium $\left(\mathrm{Ca}^{2+}\right)$, magnesium $\left(\mathrm{Mg}^{2+}\right)$, and aluminum $\left(\mathrm{Al}^{3+}\right)$, with $0.05 \mathrm{~mol} \mathrm{~L}^{-1} \mathrm{HCl}$ and $0.0125 \mathrm{~mol} \mathrm{~L}^{-1} \mathrm{H}_{2} \mathrm{SO}_{4}$ for potassium $\left(\mathrm{K}^{+}\right)$and sodium $\left(\mathrm{Na}^{+}\right)$, and with $\mathrm{pH} 70.05 \mathrm{~mol} \mathrm{~L}{ }^{-1}$ calcium acetate $\left[\mathrm{Ca}(\mathrm{OAc})_{2}\right]$ for extractable acidity $\left(\mathrm{H}^{+}+\mathrm{Al}^{+3}\right)$. Extractable $\mathrm{H}^{+}$was calculated by subtracting the tritated $\mathrm{Al}$ from extractable acidity. The $\mathrm{pH}$ in water, $1 \mathrm{~mol} \mathrm{~L}^{-1} \mathrm{KCl}$, and $0.01 \mathrm{~mol} \mathrm{~L}^{-1} \mathrm{CaCl}_{2}$ were analyzed in a 1:2.5 soil/water solution. Cation exchange capacity (CEC) was calculated from the sum of base cations $\left(\mathrm{Ca}^{2+}\right.$, $\mathrm{Mg}^{2+}, \mathrm{K}^{+}$, and $\mathrm{Na}^{+}$) plus extractable acidity. To determine mineral content, samples were ashed at $400^{\circ} \mathrm{C}$ (Embrapa 1999).

\section{Statistical Analyses}

The humic fraction C content and its percentile in relation to C_CHN were compared to each other and to the other forms of $\mathrm{C}$ using the Pearson correlation method. The relationship between the humic fractions and some soil attributes was evaluated by Pearson correlation and linear regression analyses. 
Table 1. Location, classificationa, ${ }^{a}$ and altitude of soil profiles from Brazil

\begin{tabular}{|c|c|c|c|c|}
\hline \multirow{2}{*}{$\begin{array}{l}\text { Profile } \\
\text { code }\end{array}$} & \multirow[b]{2}{*}{ Location } & \multicolumn{2}{|c|}{ Classification } & \multirow{2}{*}{$\begin{array}{l}\text { Altitude } \\
(\mathrm{m})\end{array}$} \\
\hline & & $\mathrm{SiBCS}$ & Soil taxonomy & \\
\hline AL1 & Jequiá da Praia, AL & $\begin{array}{l}\text { Organossolo Tiomórfico } \\
\text { Fíbrico térrico }\end{array}$ & Terric Sulfihemists & 3 \\
\hline AL2 & Coruripe, AL & $\begin{array}{l}\text { Organossolo Tiomórfico } \\
\text { Hêmico típico }\end{array}$ & Typic Sulfohemists & 5 \\
\hline BA2 & Ituberá, BA & $\begin{array}{l}\text { Organossolo Tiomórfico } \\
\text { Hêmico térrico }\end{array}$ & Typic Sulfohemists & 5 \\
\hline BA3 & $\begin{array}{l}\text { Arraial D'Ajuda, } \\
\text { BA }\end{array}$ & $\begin{array}{l}\text { Organossolo Tiomórfico } \\
\text { Hêmico típico }\end{array}$ & Typic Sulfohemists & 7 \\
\hline DF1 & Guará II, DF & $\begin{array}{l}\text { Organossolo Mésico } \\
\text { Sáprico típico }\end{array}$ & $\begin{array}{l}\text { Typic } \\
\text { Haplosaprists }\end{array}$ & 800 \\
\hline ES1 & Mimoso do Sul, ES & $\begin{array}{l}\text { Organossolo Mésico } \\
\text { Hêmico típico }\end{array}$ & $\begin{array}{l}\text { Hydric } \\
\text { Haplohemists }\end{array}$ & 15 \\
\hline MG1 & Juiz de Fora, MG & $\begin{array}{l}\text { Organossolo Mésico } \\
\text { Hêmico típico }\end{array}$ & $\begin{array}{l}\text { Fluvaquentic } \\
\text { Haplohemists }\end{array}$ & 874 \\
\hline MG2 & $\begin{array}{l}\text { Coronel Pacheco, } \\
\text { MG }\end{array}$ & $\begin{array}{l}\text { Organossolo Mésico } \\
\text { Hêmico térrico }\end{array}$ & $\begin{array}{l}\text { Hydric } \\
\text { Haplohemists }\end{array}$ & 432 \\
\hline MS2 & Porto Morumbi, MS & $\begin{array}{l}\text { Organossolo Tiomórfico } \\
\text { Sáprico térrico }\end{array}$ & $\begin{array}{l}\text { Terric } \\
\text { Haplosaprists }\end{array}$ & 280 \\
\hline PR2 & Tijucas do Sul, PR & $\begin{array}{l}\text { Organossolo Mésico } \\
\text { Sáprico típico }\end{array}$ & $\begin{array}{l}\text { Typic } \\
\text { Haplosaprists }\end{array}$ & 850 \\
\hline PR3 & $\begin{array}{l}\text { Serra da Baitaca, } \\
\text { PR }\end{array}$ & $\begin{array}{l}\text { Neossolo Litólico Hís- } \\
\text { tico típico }\end{array}$ & Lithic Udifolists & 1330 \\
\hline RJ1 & $\begin{array}{l}\text { Parque Nacional de } \\
\text { Itatiaia, RJ/MG }\end{array}$ & $\begin{array}{l}\text { Cambissolo Húmico } \\
\text { Distrófico típico }\end{array}$ & $\begin{array}{l}\text { Humic Pachic } \\
\text { Dystrudepts }\end{array}$ & 1700 \\
\hline RJ3 & $\begin{array}{l}\text { São José da Boa } \\
\text { Morte, RJ }\end{array}$ & $\begin{array}{l}\text { Organossolo Háplico } \\
\text { Hêmico térrico }\end{array}$ & $\begin{array}{l}\text { Hydric } \\
\text { Haplohemists }\end{array}$ & 40 \\
\hline RJ4 & Nova Friburgo, RJ & $\begin{array}{l}\text { Organossolo Mésico } \\
\text { Sáprico térrico }\end{array}$ & $\begin{array}{l}\text { Humaqueptic } \\
\text { Endoaquents }\end{array}$ & 800 \\
\hline RS3 & Cambará do Sul, RS & $\begin{array}{l}\text { Organossolo Mésico } \\
\text { Sáprico térrico }\end{array}$ & $\begin{array}{l}\text { Terric } \\
\text { Haplosaprists }\end{array}$ & 890 \\
\hline RS4 & Viamão, RS & $\begin{array}{l}\text { Organossolo Tiomórfico } \\
\text { Sáprico típico }\end{array}$ & Typic Sulfosaprists & 20 \\
\hline RS5 & Viamão, RS & $\begin{array}{l}\text { Organossolo Tiomórfico } \\
\text { Sáprico típico }\end{array}$ & Typic Sulfosaprists & 20 \\
\hline $\mathrm{SC} 2$ & $\begin{array}{l}\text { Governador Celso } \\
\text { Portela, SC }\end{array}$ & $\begin{array}{l}\text { Organossolo Tiomórfico } \\
\text { Hêmico típico }\end{array}$ & Typic Sulfohemists & 10 \\
\hline SP1 & Taubaté, SP & $\begin{array}{l}\text { Organossolo Mésico } \\
\text { Sáprico térrico }\end{array}$ & $\begin{array}{l}\text { Terric } \\
\text { Haplosaprists }\end{array}$ & 500 \\
\hline
\end{tabular}

${ }^{a}$ SiBCS (Embrapa 1999); soil taxonomy (Soil Survey Staff 1999). 
Table 2. Carbon content ${ }^{a}$ in the fraction fulvic acid (FAF), humic acid (HAF), humin (HUM), and sum of organic matter fractions, expressed as a function of soil weight and total ${ }^{b}$ organic carbon $\left(\mathrm{C}_{-} \mathrm{CHN}\right)$, and ratios of HAF/FAF and alkaline extract $(\mathrm{AE}=\mathrm{FAF}+\mathrm{HAF}) / \mathrm{HUM}$

\begin{tabular}{|c|c|c|c|c|c|c|c|c|c|c|c|}
\hline $\begin{array}{l}\text { Profile/ } \\
\text { horizons }\end{array}$ & $\begin{array}{c}\text { FAF } \\
\left(\mathrm{g} \mathrm{C} \mathrm{kg}^{-1} \text { soil }\right)\end{array}$ & $\begin{array}{c}\mathrm{HAF} \\
\left(\mathrm{g} \mathrm{C} \mathrm{kg}^{-1} \text { soil }\right)\end{array}$ & $\begin{array}{c}\text { HUM } \\
\text { ( } \mathrm{g} \mathrm{C} \mathrm{kg}^{-1} \text { soil) }\end{array}$ & $\begin{array}{c}\text { SUM } \\
\left(\mathrm{g} \mathrm{C} \mathrm{kg}^{-1} \text { soil }\right)\end{array}$ & $\begin{array}{c}\text { C_CHN } \\
\left(\mathrm{g} \mathrm{C} \mathrm{kg}^{-1} \text { soil }\right)\end{array}$ & $\begin{array}{c}\text { FAF } \\
(\% \\
\text { C_CHN })\end{array}$ & $\begin{array}{c}\text { HAF } \\
(\% \\
\text { C_CHN })\end{array}$ & $\begin{array}{c}\text { HUM } \\
(\% \\
\text { C_CHN })\end{array}$ & $\begin{array}{c}\text { SUM } \\
(\% \\
\text { C_CHN })\end{array}$ & $\begin{array}{l}\mathrm{HAF} / \\
\mathrm{FAF}\end{array}$ & $\begin{array}{l}\text { EA/ } \\
\text { HUM }\end{array}$ \\
\hline AL1 Hip1 & $39.3(6)$ & $88.5(17)$ & $172.7(11)$ & 300.6 & 357.8 & 11 & 25 & 48 & 84 & 2.25 & 0.74 \\
\hline AL1 Hip2 & $10.3(10)$ & $27.7(6)$ & $46.5(9)$ & 84.4 & 110.4 & 9 & 25 & 42 & 77 & 2.69 & 0.82 \\
\hline AL2 Haipj & 23.9 (3) & $131.0(1)$ & $156.3(16)$ & 311.2 & 305.8 & 8 & 43 & 51 & 102 & 5.48 & 0.99 \\
\hline AL2 Haij1 & $24.3(29)$ & $193.8(11)$ & $225.6(14)$ & 443.7 & 508.1 & 5 & 38 & 44 & 87 & 7.99 & 0.97 \\
\hline AL2 Haij2 & $21.2(15)$ & $139.7(4)$ & $232.7(15)$ & 393.6 & 454.7 & 5 & 31 & 51 & 87 & 6.58 & 0.69 \\
\hline AL2 Hij & $9.2(10)$ & $22.0(35)$ & $79.2(13)$ & 110.4 & 120.7 & 8 & 18 & 66 & 91 & 2.41 & 0.39 \\
\hline BA2 Haj & $23.2(14)$ & $185.7(9)$ & $252.8(7)$ & 461.7 & 525.3 & 4 & 35 & 48 & 88 & 8.01 & 0.83 \\
\hline BA2 Haij1 & $23.8(1)$ & $188.8(2)$ & $267.2(2)$ & 479.8 & 528.5 & 5 & 36 & 51 & 91 & 7.93 & 0.80 \\
\hline BA2 Haij2 & 16.7 (17) & $189.5(3)$ & $175.8(24)$ & 381.9 & 369.9 & 5 & 51 & 48 & 103 & 11.37 & 1.17 \\
\hline BA3 Haipj1 & $36.5(17)$ & $159.5(5)$ & 184.3 (19) & 380.3 & 387.6 & 9 & 41 & 48 & 98 & 4.37 & 1.06 \\
\hline BA3 Haipj2 & $16.2(29)$ & $198.9(10)$ & $281.1(6)$ & 496.2 & 555.4 & 3 & 36 & 51 & 89 & 12.31 & 0.77 \\
\hline BA3 Haij & $16.6(23)$ & 208.4 (13) & 247.2 (6) & 472.2 & 508.3 & 3 & 41 & 49 & 93 & 12.52 & 0.91 \\
\hline DF1 Hap & $11.8(8)$ & $37.8(13)$ & $38.6(7)$ & 88.2 & 101.7 & 12 & 37 & 38 & 87 & 3.21 & 1.29 \\
\hline DF1 Ha1 & $16.3(6)$ & $67.3(7)$ & $43.5(4)$ & 127.1 & 132.4 & 12 & 51 & 33 & 96 & 4.14 & 1.92 \\
\hline DF1 Ha2 & $17.8(11)$ & 119.4 (27) & $35.1(14)$ & 172.3 & 196.6 & 9 & 61 & 18 & 88 & 6.69 & 3.91 \\
\hline ES1 Hap1 & $19.1(22)$ & $47.0(13)$ & 88.0 (13) & 154.0 & 179.8 & 11 & 26 & 49 & 86 & 2.46 & 0.75 \\
\hline ES1 Нар2 & 20.1 (13) & $53.0(15)$ & 97.7 (3) & 170.7 & 157.7 & 13 & 34 & 62 & 108 & 2.63 & 0.75 \\
\hline ES1 Hai & $29.3(12)$ & $84.8(5)$ & $113.8(9)$ & 227.9 & 227.7 & 13 & 37 & 50 & 100 & 2.89 & 1.00 \\
\hline MG1 Hai & $40.0(5)$ & $65.9(12)$ & 147.4 (6) & 253.4 & 279.0 & 14 & 24 & 53 & 91 & 1.65 & 0.72 \\
\hline MG1 Ha & $23.6(35)$ & $95.5(9)$ & $77.6(5)$ & 196.7 & 213.7 & 11 & 45 & 36 & 92 & 4.05 & 1.54 \\
\hline MG1 2Hai & 34.7 (4) & $142.9(0)$ & $159.6(4)$ & 337.2 & 366.7 & 9 & 39 & 44 & 92 & 4.12 & 1.11 \\
\hline MG2 Hail & $14.0(1)$ & $45.1(21)$ & $61.1(3)$ & 120.2 & 114.8 & 12 & 39 & 53 & 105 & 3.22 & 0.97 \\
\hline MG2 Hai2 & 11.9 (18) & $68.5(10)$ & 105.7 (6) & 186.1 & 206.4 & 6 & 33 & 51 & 90 & 5.77 & 0.76 \\
\hline
\end{tabular}




\begin{tabular}{|c|c|c|c|c|c|c|c|c|c|c|c|}
\hline MS2 Haо & $20.2(5)$ & $52.3(2)$ & $41.4(2)$ & 114.0 & 121.1 & 17 & 43 & 34 & 94 & 2.58 & 1.75 \\
\hline MS2 2Ha & $7.3(28)$ & $99.4(4)$ & $79.6(4)$ & 186.3 & 189.3 & 4 & 53 & 42 & 98 & 13.66 & 1.34 \\
\hline PR2 Hap1 & $13.1(8)$ & $89.5(6)$ & $36.5(3)$ & 139.2 & 144.1 & 9 & 62 & 25 & 97 & 6.81 & 2.81 \\
\hline PR2 Hap2 & $10.0(8)$ & $143.4(10)$ & $30.7(8)$ & 184.1 & 192.8 & 5 & 74 & 16 & 95 & 14.41 & 5.00 \\
\hline PR2 Hap3 & $21.0(29)$ & 153.7 (19) & $39.6(12)$ & 214.3 & 270.0 & 8 & 57 & 15 & 79 & 7.31 & 4.42 \\
\hline PR3 Oi & $57.3(15)$ & $84.2(37)$ & $235.2(6)$ & 376.7 & 418.9 & 14 & 20 & 56 & 90 & 1.47 & 0.60 \\
\hline RJ1 A1 & $9.9(5)$ & $21.4(1)$ & $27.5(3)$ & 58.9 & 63.5 & 16 & 34 & 43 & 93 & 2.16 & 1.14 \\
\hline RJ1 A2 & $6.4(3)$ & $12.8(2)$ & $53.6(18)$ & 72.9 & 38.0 & 17 & 34 & 141 & 192 & 1.99 & 0.36 \\
\hline RJ1 Bw & $9.3(3)$ & $17.8(3)$ & $54.3(11)$ & 81.4 & 49.9 & 19 & 36 & 109 & 163 & 1.92 & 0.50 \\
\hline RJ12 Bw & $10.5(8)$ & $28.2(11)$ & $30.9(65)$ & 69.7 & 55.3 & 19 & 51 & 56 & 126 & 2.69 & 1.25 \\
\hline RJ3 Hap & 35.5 (13) & $115.3(8)$ & $131.8(6)$ & 282.6 & 339.8 & 10 & 34 & 39 & 83 & 3.25 & 1.14 \\
\hline RJ3 Hail & $42.6(14)$ & $121.2(1)$ & $139.2(16)$ & 303.0 & 314.9 & 14 & 38 & 44 & 96 & 2.85 & 1.18 \\
\hline RJ3 Hai2 & $51.0(10)$ & $104.8(4)$ & $99.7(21)$ & 255.6 & 332.3 & 15 & 32 & 30 & 77 & 2.05 & 1.56 \\
\hline RJ3 Hi & $24.0(14)$ & $95.2(12)$ & $184.4(7)$ & 303.6 & 288.5 & 8 & 33 & 64 & 105 & 3.97 & 0.65 \\
\hline RJ4 Hap1 & $15.6(10)$ & $30.5(4)$ & $20.9(3)$ & 67.1 & 91.7 & 17 & 33 & 23 & 73 & 1.95 & 2.21 \\
\hline RJ4 Hap2 & $17.2(12)$ & $34.2(16)$ & $8.9(5)$ & 60.2 & 99.5 & 17 & 34 & 9 & 61 & 1.99 & 5.79 \\
\hline RJ4 Cg1 & $12.7(20)$ & $22.4(18)$ & $3.5(5)$ & 38.6 & 69.8 & 18 & 32 & 5 & 55 & 1.76 & 9.94 \\
\hline RJ4 Cg2 & $11.0(7)$ & $22.1(10)$ & $6.0(11)$ & 39.1 & 44.8 & 25 & 49 & 13 & 87 & 2.00 & 5.56 \\
\hline RS3 Hai & $22.3(17)$ & $60.9(2)$ & $70.4(1)$ & 153.6 & 158.3 & 14 & 38 & 44 & 97 & 2.73 & 1.18 \\
\hline RS3 Ha1 & $11.0(8)$ & $52.8(4)$ & $45.2(35)$ & 109.0 & 110.3 & 10 & 48 & 41 & 99 & 4.81 & 1.41 \\
\hline RS3 Ha2 & $5.9(10)$ & $42.3(9)$ & $15.5(8)$ & 63.6 & 69.3 & 9 & 61 & 22 & 92 & 7.19 & 3.11 \\
\hline RS4 Hpj & $19.4(24)$ & $160.7(21)$ & $199.4(7)$ & 379.6 & 425.7 & 5 & 38 & 47 & 89 & 8.27 & 0.90 \\
\hline RS4 Haj & $16.6(10)$ & $153.7(2)$ & $240.1(10)$ & 410.4 & 482.2 & 3 & 32 & 50 & 85 & 9.29 & 0.71 \\
\hline RS4 Haij & $12.1(15)$ & $138.4(11)$ & $256.3(2)$ & 406.8 & 490.0 & 2 & 28 & 52 & 83 & 11.49 & 0.59 \\
\hline RS5 Hapj & $22.3(5)$ & $164.4(9)$ & $147.8(4)$ & 334.5 & 358.5 & 6 & 46 & 41 & 93 & 7.38 & 1.26 \\
\hline RS5 Haj & $24.4(4)$ & $134.0(7)$ & $111.4(2)$ & 269.9 & 291.6 & 8 & 46 & 38 & 93 & 5.48 & 1.42 \\
\hline SC2 Haij1 & 30.9 (13) & $143.1(9)$ & $235.6(9)$ & 409.6 & 460.0 & 7 & 31 & 51 & 89 & 4.63 & 0.74 \\
\hline
\end{tabular}


Table 2. Continued

\begin{tabular}{|c|c|c|c|c|c|c|c|c|c|c|c|}
\hline $\begin{array}{l}\text { Profile/ } \\
\text { horizons }\end{array}$ & $\begin{array}{c}\text { FAF } \\
\left(\mathrm{g} \mathrm{C} \mathrm{kg}^{-1} \text { soil }\right)\end{array}$ & $\begin{array}{c}\mathrm{HAF} \\
\left(\mathrm{g} \mathrm{C} \mathrm{kg}^{-1} \text { soil) }\right.\end{array}$ & $\begin{array}{c}\text { HUM } \\
\left(\mathrm{g} \mathrm{C} \mathrm{kg}^{-1} \text { soil) }\right.\end{array}$ & $\begin{array}{c}\text { SUM } \\
\left(\mathrm{g} \mathrm{C} \mathrm{kg}^{-1} \text { soil) }\right.\end{array}$ & $\begin{array}{c}\mathrm{C}_{-} \mathrm{CHN} \\
\left(\mathrm{g} \mathrm{C} \mathrm{kg}^{-1} \text { soil) }\right.\end{array}$ & $\begin{array}{c}\text { FAF } \\
(\% \\
\text { C_CHN })\end{array}$ & $\begin{array}{c}\text { HAF } \\
(\% \\
\text { C_CHN) }\end{array}$ & $\begin{array}{c}\text { HUM } \\
(\% \\
\text { C_CHN) }\end{array}$ & $\begin{array}{c}\text { SUM } \\
(\% \\
\text { C_CHN })\end{array}$ & $\begin{array}{l}\mathrm{HAF} / \\
\mathrm{FAF}\end{array}$ & $\begin{array}{l}\text { EA/ } \\
\text { HUM }\end{array}$ \\
\hline SC2 Haij2 & $24.5(8)$ & $188.4(6)$ & $260.3(7)$ & 473.2 & 528.1 & 5 & 36 & 49 & 90 & 7.68 & 0.82 \\
\hline SP1 Hp1 & $23.7(12)$ & $112.8(13)$ & $53.7(8)$ & 190.2 & 207.4 & 11 & 54 & 26 & 92 & 4.76 & 2.54 \\
\hline SP1 Hp2 & $13.2(3)$ & $95.1(6)$ & $17.7(14)$ & 126.0 & 144.4 & 9 & 66 & 12 & 87 & 7.19 & 6.12 \\
\hline Average & 20.8 & 99.2 & 116.4 & 236.3 & 259.6 & 10 & 40 & 44 & 94 & 5.31 & 1.84 \\
\hline $\begin{array}{l}\text { Standard } \\
\text { deviation }\end{array}$ & 11.2 & 57.8 & 85.6 & 140.2 & 158.4 & 5 & 12 & 22 & 20 & 3.42 & 1.81 \\
\hline
\end{tabular}

${ }^{a}$ Average value of triplicates (variation coefficient, $\%$, between brackets)

${ }^{b}$ C_CHN method = total C, Perkin Elmer 2400 CHNS analyzer. 


\section{RESULTS AND DISCUSSION}

\section{Carbon Distribution in the Different Soil Humic Fractions}

The humic substance fractionating resulted in good reproducibility and consistent data considering the complexity of the process. Twenty-six samples ( $49 \%$ of total) presented variation coefficients less than $10 \%, 45$ samples $(85 \%)$ had coefficient of variation less than $20 \%$, and only 1 sample showed variation coefficients more than $30 \%$ (Table 2). For HAF, 31 samples $(58 \%$ of total) presented variation coefficients less than $10 \%$, 48 samples $(90 \%)$ showed variation coefficients less than $20 \%$, and only 2 samples had variation coefficients more than $30 \%$ (Table 2). In HUM, 34 samples (64\% of the total) presented variation coefficients less than $10 \%, 49$ samples $(92 \%)$ with a variation coefficient less than $20 \%$, and only 2 had variation coefficients more than $30 \%$ (Table 2). Variability of the humic fractions per sample did not show a relationship with the content of organic matter, because the variation coefficient was not correlated to the $\mathrm{C}$ content.

Considering the average values of the three organic-matter fractionating repetitions, the humin fraction presented the highest average and standard deviation $\left(116.4 \pm 85.5 \mathrm{~g} \mathrm{~kg}^{-1}\right)$. This fraction was predominant in most soil samples, with an average value of $43.8 \%$ of the total $\mathrm{C}$ determined by CHN (C_CHN). However, it showed a high variation coefficient $(74 \%)$ and high amplitude, with minimum and maximum values of 3.5 and $281.1 \mathrm{~g} \mathrm{~kg}^{-1}$, respectively. The method used tends to overestimate values of true humic substances, because it quantifies nonsoluble $\mathrm{C}$ forms in alkaline medium, which includes fibers and other forms of organic matter such as the light organic matter (Benites 1998).

The FAF fraction consists of true fulvic acids as well as other lowmolecular-weight organic compounds, co-extracted in the extraction progress (Benites 1998). The FAF presented the lowest values of average and standard deviation $\left(20.8 \pm 11.2 \mathrm{~g} \mathrm{~kg}^{-1}\right)$, representing $10 \%$ of the total $\mathrm{C}$ on average (Table 2), and also the lowest variability, with variation coefficient of $54 \%$ [with a minimum HUM value $\left(5.9 \mathrm{~g} \mathrm{~kg}^{-1}\right)$ and maximum $\left(57.3 \mathrm{~g} \mathrm{~kg}^{-1}\right)$ content]. The (HAF) exhibited average value and standard deviation of $99.2 \pm 57.9 \mathrm{~g} \mathrm{~kg}^{-1}$, with variation coefficient of $58 \%$, and represents $40 \%$ of the total $C$ on average. The minimum value was also above the HUM minimum value $\left(12.5 \mathrm{~g} \mathrm{~kg}^{-1}\right)$ and maximum value of $208.4 \mathrm{~g} \mathrm{~kg}^{-1}$ (Table 2).

From a total of 53 samples collected in this study, 3 showed FAF values compared to HUM. This occurred in horizons with relatively low organic C content, near the minimum limit to be identified as organic horizon and with low fiber content or from mineral horizons, corresponding to horizons $\mathrm{Ha}$, $\mathrm{Cg} 1$, and $\mathrm{Cg} 2$ of a hydromorphic soil from Nova Friburgo region, Rio de Janeiro State, profile RJ4. In 20 samples, C content in HAF was higher than that found in HUM fraction, and most of these horizons contained low fiber. 


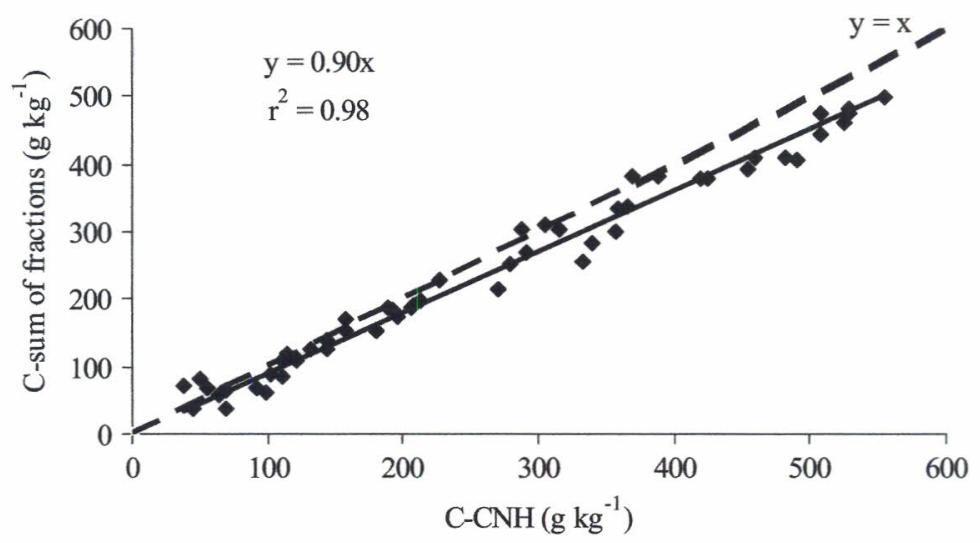

Figure 1. Relationship between the sum of humic fractions and total carbon measured by the CHN reference method. Coefficient significant at 0.001 .

The sum of the humic fractions averaged $93.9 \%$ of the total $\mathrm{C}$ determined by $\mathrm{CHN}$, dry combustion as a reference (Table 2). These variables were also highly correlated (Figure 1).

The recovery percentile showed a large variation, ranging from 55.3 to $191.8 \%$. However, the recovery factor had a non uniform distribution (Figure 2), and there was a higher recovery variability in relatively low total $\mathrm{C}$ content samples, below $120 \mathrm{~g} \mathrm{~kg}^{-1}$, where the variation coefficient is of $39 \%$ and the maximum and minimum values are within this range. The samples with high organic $\mathrm{C}$ presented low variability, with variation coefficient equal to $7 \%$ and minimum and maximum values of 76.9 and

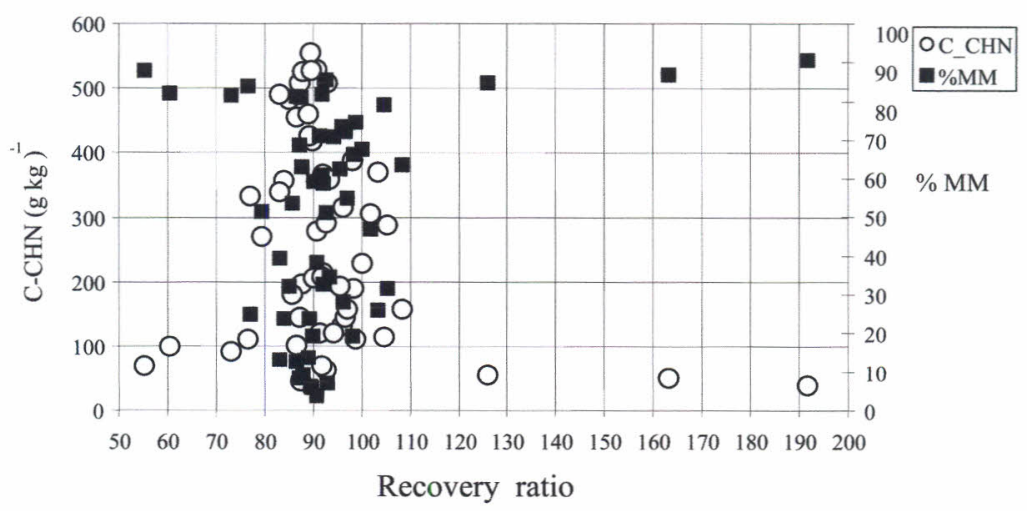

Figure 2. Relationship between carbon recovery ratio, soil mineral material (MM) percentage, and total $\mathrm{C}$ measured by the $\mathrm{CHN}$ reference method. 
$108.3 \%$, respectively. The highest variability in both recovery forms was verified for low organic $\mathrm{C}$ values and higher mineral material content, profiles RJ1 and RJ4 (Figure 2).

For samples with mineral material (\%MM) of more than $80 \%$ (Figure 2 ), a similar effect on variability was observed. These data demonstrate lower result reproducibility in samples with total $\mathrm{C}$ contents less than $120 \mathrm{~g} \mathrm{~kg}^{-1}$ or \% MM more than $80 \%$. When observed with the simple linear regression analysis, with a confidence interval of $95 \%$, total carbon as the $\mathrm{X}$ axis and the sum of humic fractions as $\mathrm{Y}$ axis, the linear coefficient may be considered as 0 (zero) and the angular coefficient has as minimum, average, and maximum values $0.88,0.90$, and 0.92 , respectively, indicating that the sum of humic fractions represents from 88 to $92 \%$ of total C obtained from $\mathrm{CHN}$ reference method (Figure 1).

The values of the ratio HAF/FAF ranged from 1.47 to 14.41 , with a predominance of HAF in all soil samples. According to INCORA (1974), a higher value for HAF/FAF indicates higher polymerization degree of organic matter. The increase in this ratio is observed in most profiles without regard to soil depth, which is the inverse of what occurs in mineral soils (Benites, Ker and Mendonga 2000). These values result from the fact that Histosol upper horizons periodically are placed under oxidizing conditions, due to watertable seasonal oscillation. This furthers the formation of soluble organic compounds as a result of an increase in the microbiological activity. Zech, Guggemberger, and Schulten (1994) observed highest formation of lowermolecular-weight humic substances in soils during hotter periods and with higher microbiological activity.

The ratio $\mathrm{AE} / \mathrm{HUM}$, where $\mathrm{AE}$ is the sum of the FAF and HAF fractions, also showed a large variation, with values between 0.36 and 9.94. However, $79 \%$ of samples gave values lower than 2 . According to Benites, Ker, and Mendonga (2000), this ratio provides information on the soil genesis, identifying movement zones or $\mathrm{C}$ accumulation and identifying peaks of the $\mathrm{AE} /$ HUM ratio in spodic horizons.

The profile RJ4, which is classified as a Humaqueptic Endoaquents, formed under hydromorphic conditions with high mineral material contents and under coverage of pasture, presents a behavior similar to that observed in Spodosols (Benites et al. 2001). In this profile, a peak of the relation $\mathrm{AE} / \mathrm{HUM}$ occurred in the third horizon $(\mathrm{Cg} 1)$, which coincides with the increase in the mineral material percentage, thus demonstrating high interaction of the soluble alkaline humic fractions with the soil mineral matrix. The profile PR3, formed by a single $\mathrm{O}$ horizon directly overlying rock, located in a mountainous topography and well drained, presented a low $\mathrm{AE} / \mathrm{HUM}$ ratio, indicating the loss of more soluble humic fractions and maintenance of insoluble fractions. The other profiles vary in behavior with soil local environment, independent of soil depth. Thus, the AE/HUM variations in Histosols indicate that the dynamics of humic substances is different from mineral soils. 


\section{Humic Fractions and Carbon and Nitrogen}

The total $\mathrm{C}$ values determined through $\mathrm{C} \_\mathrm{CHN}$ showed significant correlation at $0.1 \%$ probability with fractions FAH $(r=0.90)$ and HUM $(r=0.95)$, although there was no significant correlation at this level with FAF. According to Schnitzer (1986), the humic acids in association with colloids form insoluble complexes at $\mathrm{pH}$ lower than 6.5 , thus enabling the immobilization and accumulation of this fraction in acid soils. The humin fraction, as determined, includes undecomposed fibers present in the soil organic matter, which should be responsible for the high correlation observed between this fraction and the total $\mathrm{C}$. The lower correlation of FAF with the total organic $\mathrm{C}$ reflects the higher FAF solubility and mobility in the soil (Stevenson 1994).

The amount of total $\mathrm{N}$ measured by the $\mathrm{CHN}$ method showed a high correlation with the FAF fraction $(\mathrm{r}=0.86, \mathrm{P}=0.001)$ (Figure 3), indicating that this fraction is closely related to the soil $\mathrm{N}$ content. The FAH $(r=0.53)$, HUM $(r=0.66)$, and the sum of humic fractions $(r=0.69)$ also correlated with the $\mathrm{N}$ level and the coefficients are significant at $\mathrm{P}=0.01$.

The values obtained in the alkaline extract readings at $380 \mathrm{~nm}$ showed high correlation with C_CHN contents, indicating the potential of the method to estimate the total $\mathrm{C}$ content in Histosols and related soils with high organic-matter content. Similarly, the alkaline extract readings at $465 \mathrm{~nm}$ were correlated with FAH (Table 3). The readings at $665 \mathrm{~nm}$ did not have significant correlations with other properties.

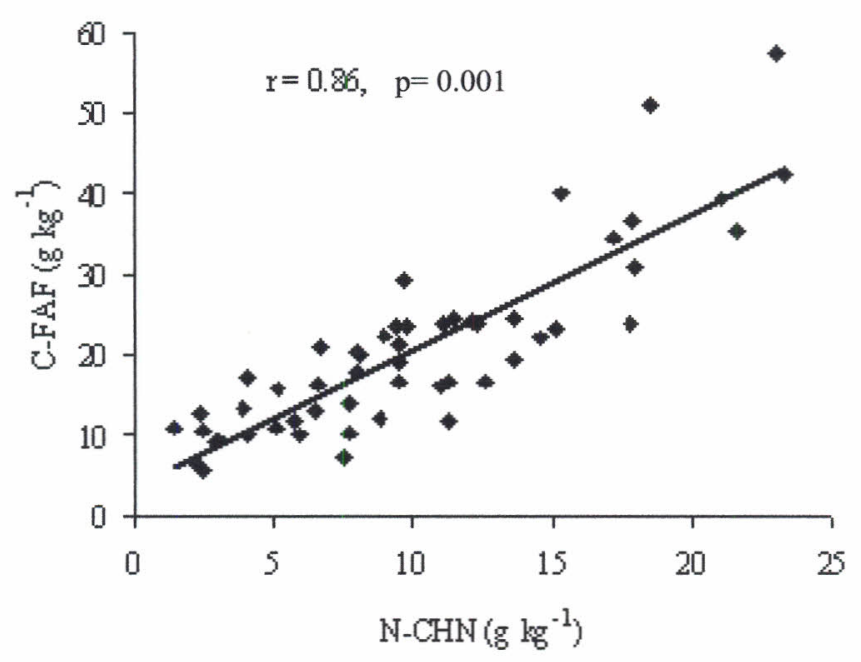

Figure 3. Relationship between content of $\mathrm{N}$ measured by the $\mathrm{CHN}$ reference method with fulvic acid fraction (FAF). 
Table 3. Matrix with correlation coefficients for organic fractions FAF, HAF, HUM, and total C_CHN, as related to CEC, sum of bases, phosphorus, exchangeable acidity, extractable $\mathrm{Al} ; \mathrm{pH}$ in water, $\mathrm{KCl}$, and $\mathrm{CaCl}_{2}$; and bulk density and alkaline extract measured at 380,465 and $665 \mathrm{~nm}$

\begin{tabular}{|c|c|c|c|c|}
\hline Property & FAF & HAF & HUM & C_CHN \\
\hline CEC & $0.01 \mathrm{~ns}$ & $0.60^{* * *}$ & $0.44^{* *}$ & $0.50^{* * *}$ \\
\hline Sum of bases & $0.42^{* *}$ & $0.28^{*}$ & $0.28^{*}$ & $0.33^{*}$ \\
\hline $\mathrm{P}$ & $0.49^{* * *}$ & $-0.08 \mathrm{~ns}$ & $0.15 \mathrm{~ns}$ & $0.09 \mathrm{~ns}$ \\
\hline $\mathrm{H}^{+}$ & $-0.11 \mathrm{~ns}$ & $0.66^{* * *}$ & $0.42^{* *}$ & $0.50^{* * *}$ \\
\hline $\mathrm{Al}^{3+}$ & $-0.13 n s$ & $-0.05 \mathrm{~ns}$ & $0.05 \mathrm{~ns}$ & $-0.01 \mathrm{~ns}$ \\
\hline $\mathrm{pH} \mathrm{KCl}$ & $-0.18 n s$ & $-0.73^{* * *}$ & $-0.65^{* * *}$ & $-0.71^{* * *}$ \\
\hline $\mathrm{pH}$ water & $-0.03 n s$ & $-0.58^{* * *}$ & $-0.60^{* * *}$ & $-0.58^{* * *}$ \\
\hline $\mathrm{pH} \mathrm{CaCl}{ }_{2}$ & $-0.02 \mathrm{~ns}$ & $-0.50^{* * *}$ & $-0.63^{* * *}$ & $-0.56^{* * *}$ \\
\hline $\begin{array}{l}\text { Abs. at } \\
\quad 380 \mathrm{~nm}\end{array}$ & $0.41^{* *}$ & $0.89^{* * *}$ & $0.87^{* * *}$ & $0.92^{* * *}$ \\
\hline $\begin{array}{l}\text { Abs. at } \\
\quad 465 \mathrm{~nm}\end{array}$ & $0.28^{*}$ & $0.92^{* * *}$ & $0.77^{* * *}$ & $0.86^{* * *}$ \\
\hline $\begin{array}{l}\text { Abs. at } \\
\quad 665 \mathrm{~nm}\end{array}$ & $-0.09 \mathrm{~ns}$ & $0.50^{* * *}$ & $0.12 \mathrm{~ns}$ & $0.29^{*}$ \\
\hline Ds & $-0.55^{* * *}$ & $-0.63^{* * *}$ & $-0.71^{* * *}$ & $-0.75^{* * *}$ \\
\hline
\end{tabular}

***Significant at $0.1 \% ;{ }^{* *}$ significant at $1 \%$; ${ }^{*}$ significant at $5 \%$; ns: non significant. Notes: $\mathrm{FAF}=$ fulvic acid fraction; $\mathrm{HAF}=$ humic acid fraction; $\mathrm{HUM}=$ humin; $\mathrm{CEC}=$ cation exchange capacity; sum of bases $=(\mathrm{Ca}+\mathrm{Mg}+\mathrm{Na}+\mathrm{K})$; Ds $=$ soil bulk density. C_CHN method = total C, Perkin Elmer 2400 CHNS analyzer.

\section{Humic Fractions and Soil Attributes}

The CEC presented positive significant correlation with HAF, HUM, and total C_CHN. Related to soil acidity, the strongest correlation was obtained for HAF, $\mathrm{H}^{+}$, and $\mathrm{pH}$ in $\mathrm{KCl}$ (Table 3). This result indicates the important role of the humic acid fraction in the generation of negative charges in soil, thus contributing to CEC. Similar results were obtained by other authors on different tropical soil classes (Benites 1998; Benites, Ker, and Mendonga 2000; Souza et al. 2003). The sum of bases and phosphorous (P) content presented a significant correlation with FAF, indicating FAF is more related to the level of nutrients available in soil. FAF presented no correlation with acidity indicators $\mathrm{H}^{+}$, $\mathrm{Al}^{3+}$, and $\mathrm{pH}$. These results suggest that in Histosols with higher natural fertility, the transformation of the organic matter favors the FAF production. The highest correlation coefficients in absolute value for $\mathrm{pH}$ were found with $\mathrm{pH}$ in $\mathrm{KCl}$ and variables HAF, HUM, and C_CHN (Table 3).

The $\mathrm{C} / \mathrm{N}$ ratio did not show a significant correlation with any of the humic fractions. However, it is possible to define an equation with a determination coefficient $\mathrm{r}^{2}$ of $0.594(\mathrm{P}<0.001$ ) with the use of the multiple regression analysis, where the $\mathrm{C} / \mathrm{N}$ ratio may be determined as a function of the FAF 
content and the sum of humic fractions (SUM), where all coefficients are significant at $\mathrm{P}=0.001$ :

$$
\frac{\mathrm{C}}{\mathrm{N}}=23.454-0.593(\mathrm{FAF})+0.065(\mathrm{SUM}) \text {. }
$$

The soil bulk density (Ds) showed negative significant correlation with C_CHN and with all humic fractions. That is, the higher organic-matter content resulted in lower Ds, therefore a higher subsidence potential.

\section{CONCLUSIONS}

The method employed for humic substance fractionation was shown to be suitable, giving good reproducibility and easy technical execution, which indicates it may be included in routine laboratory characterization of Histosols and related soils with high organic-matter content.

The FAF was better correlated with level of nutrients than with soil acidity variables and thus may be an indicator of nutrient availability on Histosols. The humic acid and humin fractions presented high correlation with CEC, active acidity $\left(\mathrm{H}^{+}\right)$, and $\mathrm{pH}$. The humin fraction and the alkaline extract absorbance level measured at $380 \mathrm{~nm}$ and $465 \mathrm{~nm}$ showed a high correlation with the total soil organic $\mathrm{C}$ content.

\section{ACKNOWLEDGMENT}

We are grateful to CAPES, CNPq, and FAPERJ for the financial support for this project.

\section{REFERENCES}

Benites, V.M. (1998) Caracterização química e espectroscópica da matéria orgânica e suas relações com a gênese de solos da Serra do Brigadeiro, Zona da Mata mineira, Dissertação (Mestrado em Solos e Nutrição de Plantas). Universidade Federal de Viçosa: Viçosa, Brazil.

Benites, V.M., Schaefer, C.E.R.G., Mendonça, E.S., and Martin Neto, L. (2001) Caracterização da matéria orgânica e micromorfologia de solos sob Campos de Altitude no Parque Estadual da Serra do Brigadeiro. Revista Brasileira de Ciência do Solo, 25: 661-674.

Benites, V.M., Ker, J.C., and Mendonça, E.S. (2000) Fracionamento quantitativo de substâncias húmicas como auxiliar na identificação de diferentes solos da região Sul do Brasil-VI RCC, 184-192. In Guia de Excursão de Estudos de Solos nos Estados do Rio Grande do Sul, Santa Catarina e Paraná; EMBRAPA Florestas: Colombo, Paraná, Brazil

Benites, V.M., Madari, B., and Machado, P.L.O.A. (2003) Extração e fracionamento quantitativo de substâncias húmicas do solo: um procedimento simplificado de baixo custo. Comunicado técnico 16; Embrapa Solos: Rio de Janeiro, Brazil. 
Embrapa, Centro Nacional de Pesquisa de Solos. (1997) Manual de metodos de analises de solos, 212, Rio de Janeiro, Brazil, Embrapa Solos.

Embrapa, Centro Nacional de Pesquisa de Solos. (1999) Sistema brasileiro de classificação de solos; Brasília: Embrapa Produção de Informação, 412, Embrapa Solos: Rio de Janeiro, Brazil.

Ferreira, J.A., Martin-Neto, L., Vaza, C.M.P., and Regitano, J.B. (2002) Organic compounds in the environment sorption interactions between imazaquin and a humic acid extracted from a typical Brazilian Oxisol. Journal of Environmental Quality, 31: 1665-1670.

INCORA-Instituto Colombiano de la Reforma Agraria. (1974) Mapificacion caracterizacion $Y$ clasificacion de los suelos organicos del Valle de Sibundoy; INCORA: Bogotá.

Jones, K.D. and Huang, W.H. (2003) Evaluation of toxicity of the pesticides, chlorpyrifos, and arsenic, in the presence of compost humic substances in aqueous systems. Journal of Hazardous Material, 103 (1-2): 93-105.

Lal, R. (2004) Soil carbon sequestration to mitigate climate change. Geoderma, 123: $1-22$.

Lemos, R.C. and Santos, R.D. (1996) Manual de descrição e coleta de solos no campo, 3rd ed.; SBCS/EMBRAPA-CNPS: Campinas, Brazil

MacCarthy, P. (2001) The principles of humic substances. Soil Science, 166: 738-751.

McBride, M.B. (1994) Environmental Chemistry of Soils; Oxford University Press: New York.

Nelson, D.W. and Sommers, L.E. (1982) Total carbon, organic carbon, and organic matter. American Society of Agronomy Journal, 9: 539-577.

Schnitzer, M. and Khan, S.U. (1978) Soil organic matter; Elsevier: New York

Schnitzer, M. (1986) Binding of humic substances by soil mineral colloids. In Interactions of Soil Minerals with Natural Organics and Microbes; Huang, P.M. and and Schnitzer, M. (eds.), SSSA: Madison, Wisc., 77-101.

Soil Survey Division Staff. (1993). Soil Survey Manual; USDA-SCS Agric. Handb, 18). U.S. Government Printing Office: Washington, D.C.

Soil Survey Staff. (1999) Soil Taxonomy: A Basic System of Soil Classification for Making and Interpreting Soil Surveys; USDA-SCS Agric.Handb. 436, U.S. Government Printing Office: Washington, D.C.

Souza, L.F., Madari, B., Benites, V.M., Cunha, T.J.F., and Neves, E.G. (2003) Relação entre a fertilidade e as substâncias húmicas em uma terra preta na Amazônia. In Anais do XXIX Congresso Brasileiro de Ciência do Solo (CD-ROM); São Paulo, Ribeirão Preto, Brazil.

Stevenson, F.J. (1994) Humus Chemistry: Genesis, Composition, Reactions, 2nd ed.; John Wiley and Sons: New York.

Tombácz, E. and Meleg, E. (1990) A theoretical explanation of the aggregation of humic substances as a function of $\mathrm{pH}$ and electrolyte concentration. Organic Geochemistry, 15: 375-381.

Yeomans, J.C. and Bremner, J.M. (1998) A rapid and precise method for routine determination of organic carbon in soil. Communications in Soil Science and Plant Analysis, 19: 1467-1476.

Zech, W., Guggemberger, G., and Schulten, H.R. (1994) Budgets and chemistry of dissolved organic carbon in forest soils: Effect of anthropogenic soil acidification. Science of Total Environment, 152: 49-62. 